\title{
Physical Layer Security for Cooperative Relaying Over Generalized-K Fading Channels
}

\author{
Ling Wu, Liang Yang, Jianchao Chen, and Mohamed-Slim Alouini, Fellow, IEEE
}

\begin{abstract}
In this letter, we analyze the secrecy performance for a cooperative diversity system with amplify-and-forward (AF) over generalized-K fading channels. More specifically, some lower bounds on the secrecy performance, such as secure outage probability (SOP), average secrecy capacity (ASC), and strictly positive secrecy capacity (SPSC), are derived in closed-form. Finally, we provide simulation results to verify the accuracy of our analytical results.
\end{abstract}

Index Terms-Amplify-and-forward, average secrecy capacity, physical layer security, secure outage probability, strictly positive secrecy capacity.

\section{INTRODUCTION}

Recently, physical layer security which is a new method to solve the information security problem in the wireless communication system has aroused widespread concern, and has a wide range of research and application prospects [1]. Further, as stated in [2], cooperative diversity can be exploited to enhance the secrecy performance [3]-[6], for instance, optimal relay selection [3], the technology of multiuser cooperative relay [4], different diversity combining techniques [5], incremental hybrid decode-amplify-forward (IHDAF) scheme with transmit antenna selection (TAS) [6]. However, most of the analytical results are only available to the Rayleigh fading channels.

Recently, secrecy performance analysis for some complicated channel models has been presented in [7]-[9], for instance, log-normal fading [7], Weibull fading [8], and Rician fading [9]. However, in some practical environments, a wireless communication system maybe suffers from both the large-scale fading and small-scale fading. For such a case, a composite model called the generalized-K $\left(K_{G}\right)$ distribution can be used to characterize the statistic behavior [10]. Until now, regarding the $K_{G}$ distribution model, there are many related works focusing on the performance analysis for different system settings. However, the secrecy performance over

Copyright (c) 2015 IEEE. Personal use of this material is permitted. However, permission to use this material for any other purposes must be obtained from the IEEE by sending a request to pubs-permissions@ieee.org.

Manuscript received Dec 28, 2017; revised Jan 30, 2018; accepted Jan 31, 2018. This work was in part supported by the National Natural Science Foundation of China (NSFC) under Grant 61671160, the Department of Education of Guangdong Province (No. 2016KZDXM050), and the open research fund of National Mobile Communications Research Laboratory, Southeast University (No. 2018D01). The review of this paper was coordinated by Prof. Justin Coon.

L. Wu, L. Yang, and J. Chen are with the School of Information Engineering, Guangdong University of Technology, Guangzhou, China.

M.-S. Alouini is with the Computer, Electrical, and Mathematical Science and Engineering Division, King Abdullah University of Science and Technology, Thuwal, Saudi Arabia.
$K_{G}$ fading channels is still lack of sufficient consideration. More recently, assuming $K_{G}$ fading channels, the authors in [11] considered the secrecy performance for a single-input multiple-output (SIMO) system, the average secrecy capacity (ASC), strictly positive secrecy capacity (SPSC), and secure outage probability (SOP) were derived. By using a mixture gamma distribution, secrecy performance over $K_{G}$ fading channels was analyzed in [12].

To the best of the authors' knowledge, the secrecy performance analysis for a relaying system over $K_{G}$ fading channels is still not reported in the literature. In this letter, our intention is to analyze the secrecy performance for amplify-and-forward (AF) relaying networks suffering from an eavesdropper over $K_{G}$ fading channels. We derive some analytical expressions for the SOP, SPSC, and ASC, respectively.

\section{SYSTEM AND CHANNEL MODELS}

Consider an amplify-and-forward (AF) relay system consisting of source $(S)$, destination $(D)$, one relay $(R)$, and an eavesdropper $(E)$, where all the nodes have single antennas. We assume that a half-duplex relaying operation and there is no direct link between $S$ and $D$ due to obstacles or deep shadowing. We further assume that $E$ can receive signals from both $S$ and $R$. Furthermore, we assume that the channel state information (CSI) of the $R-D$ and $R-E$ links is available to $R$. At the first time slot, $S$ broadcasts $s_{1}$ with unit power to $R$ and $E$, respectively. Therefore, the received signals at $R$ and $E$ can be written as

$$
y_{S x}=h_{1 x} s_{1}+\omega_{1 x}
$$

where $h_{1 x}(x \in\{R, E\})$ denotes the channel gain of $S-x$ link, and $\omega_{1 x}$ is an additive white Gaussian noises (AWGN) with variance $N_{0}$. In the second phase, $y_{S R}$ is amplified and forwarded to $D$ and $E$, respectively. Then, the received signal at $D$ is

$$
y_{R y}=h_{2 y} G\left(h_{1 R} s_{1}+\omega_{1 R}\right)+\omega_{2 y},
$$

where $h_{2 y}(y \in\{D, E\})$ denotes the channel gain between $R$ and $y$, and $\omega_{2 y}$ is the AWGN with variance $N_{0}$. Assuming CSI-assisted relaying, then the amplifying factor at the relay is $G=\sqrt{\operatorname{Pr} /\left(\left|h_{1 R}\right|^{2}+N_{0}\right)}$ with transmit power $\operatorname{Pr}$ at $R$.

From (1) and (2), the instantaneous signal-to-noise ratio (SNRs) at $D$ and $E$ can be written as $\gamma_{D}=\frac{\gamma_{1} \gamma_{2}}{1+\gamma_{1}+\gamma_{2}}$ and $\gamma_{E}=$ $\gamma_{4}+\frac{\gamma_{1} \gamma_{3}}{1+\gamma_{1}+\gamma_{3}}$, respectively, where $\gamma_{i}(i \in\{1,2,3,4\})$ denote the SNRs of $S-R, R-D, R-E$, and $S-E$ links, respectively. Specially, at high SNR, $\gamma_{D} \approx \gamma_{1} \gamma_{2} /\left(\gamma_{1}+\gamma_{2}\right) \leq \min \left\{\gamma_{1}, \gamma_{2}\right\}$, $\gamma_{E} \approx \gamma_{4}+\gamma_{1} \gamma_{3} /\left(\gamma_{1}+\gamma_{3}\right) \leq \gamma_{4}+\min \left\{\gamma_{1}, \gamma_{3}\right\}$. We assume that all channels suffer from independent and not necessarily 
identically distributed quasi-static $K_{G}$ fading. According to [11], the probability density function (PDF) of $\gamma_{i}$ can be shown to be given by

$$
f_{\gamma_{i}}(\gamma)=\frac{2 \Xi_{i}^{\frac{k_{i}+m_{i}}{2}}}{\Gamma\left(m_{i}\right) \Gamma\left(k_{i}\right)} \gamma^{\frac{k_{i}+m_{i}-2}{2}} K_{k_{i}-m_{i}}\left(2\left(\Xi_{i} \gamma\right)^{\frac{1}{2}}\right),
$$

where $K_{v}(\cdot)$ is the modified Bessel function of the second kind with order $v$ [14, Eq. (8.407.1)], $\Gamma(z)$ is the gamma function, $\Xi_{i}=k_{i} m_{i} / \bar{\gamma}_{i}, k_{i}$ and $m_{i}$ are the fading parameters, and $\bar{\gamma}_{i}$ is the average value of $\gamma_{i}$. In [11], the $K_{v}(\cdot)$ is expressed in terms of the Meijer's G-function and then all the analytical results for the secrecy performance are obtained in terms of the Meijer's G-functions. For tractable analysis, similar to [12][13], we utilize an approximate method to model the $K_{G}$ fading. Then, the PDF expression (3) can be rewritten as

$$
f_{\gamma_{i}}(\gamma)=\sum_{j=1}^{L} a_{i, j} \gamma^{m_{i}-1} \exp \left(-\zeta_{i, j} \gamma\right)
$$

where $a_{i, j}=\frac{\theta_{i, j}}{\sum_{v=1}^{L} \theta_{i, v} \Gamma\left(m_{i}\right) \zeta_{i, v}^{-m_{i}}}, \quad \zeta_{i, j}=\frac{\Xi_{i}}{t_{j}}, \theta_{i, j}=$ $\frac{\Xi_{i}^{m_{i}} \omega_{j} t_{j}^{k_{i}-m_{i}-1}}{\Gamma\left(k_{i}\right) \Gamma\left(m_{i}\right)}, t_{j}$ and $\omega_{j}$ are the abscissas and the factor for the Gaussian-Laguerre integration, and $L$ is the number of terms.

Assuming $m_{i}$ is integer and applying [14, Eq. (3.381.1)] and [14, Eq. (8.352.1)], we can express the cumulative distribution function $(\mathrm{CDF})$ of $\gamma_{i}$ as [12]

$$
F_{\gamma_{i}}(\gamma)=\sum_{j=1}^{L} A_{i, j}\left(1-\sum_{p=0}^{m_{i}-1} \frac{\zeta_{i, j}^{p}}{p !} \gamma^{p} \exp \left(-\zeta_{i, j} \gamma\right)\right),
$$

where $A_{i, j}=\left(m_{i}-1\right) ! a_{i, j} \zeta_{i, j}^{-m_{i}}$

\section{SECRECY PERFORMANCE ANALYSIS}

From [15], the instantaneous secrecy capacity is given by

$$
C_{s}\left(\gamma_{D}, \gamma_{E}\right)=\max \left\{\frac{1}{2} \ln \left(1+\gamma_{D}\right)-\frac{1}{2} \ln \left(1+\gamma_{E}\right), 0\right\} \text {, }
$$

where $\ln \left(1+\gamma_{D}\right)$ and $\ln \left(1+\gamma_{E}\right)$ correspond to the data rates of the main channel and the eavesdropping links, respectively.

A. Secure Outage Probability

The SOP can be formulated as

$$
\begin{aligned}
& \mathrm{SOP}=\operatorname{Pr}\left(\mathrm{C}_{\mathrm{s}}\left(\gamma_{\mathrm{D}}, \gamma_{\mathrm{E}}\right)<\mathrm{R}_{\mathrm{th}}\right) \\
& =1-\operatorname{Pr}\left(1+\min \left\{\gamma_{1}, \gamma_{2}\right\}>\mathrm{C}_{\mathrm{th}}\left(1+\gamma_{4}+\min \left\{\gamma_{1}, \gamma_{3}\right\}\right)\right) \\
& =1-\left[\operatorname{Pr}\left(1+\min \left\{\gamma_{1}, \gamma_{2}\right\}>\mathrm{C}_{\mathrm{th}}\left(1+\gamma_{4}+\gamma_{1}\right), \gamma_{1}<\gamma_{3}\right)\right. \\
& \left.+\operatorname{Pr}\left(1+\min \left\{\gamma_{1}, \gamma_{2}\right\}>\mathrm{C}_{\mathrm{th}}\left(1+\gamma_{4}+\gamma_{3}\right), \gamma_{1}>\gamma_{3}\right)\right],
\end{aligned}
$$

where $R_{t h}\left(R_{t h} \geq 0\right)$ is the target rate and $C_{t h}=$ $\exp \left(2 R_{t h}\right) \geq 1$. From (7), for $\gamma_{1}<\gamma_{2}$ and $\gamma_{1}<\gamma_{3}$, both $1+\gamma_{1}>C_{t h}\left(1+\gamma_{1}+\gamma_{4}\right)$ and $1+\gamma_{1}>C_{t h}\left(1+\gamma_{3}+\gamma_{4}\right)$ are impossible events. On the other hand, $\gamma_{1}>\gamma_{2}$ and $\gamma_{1}<\gamma_{3}$, $1+\gamma_{2}>C_{t h}\left(1+\gamma_{1}+\gamma_{4}\right)$ and $1+\gamma_{2}>C_{t h}\left(1+\gamma_{3}+\gamma_{4}\right)$ are also impossible events. Then, we readily have

$$
\begin{aligned}
\mathrm{SOP} & =1-\operatorname{Pr}\left(1+\min \left\{\gamma_{1}, \gamma_{2}\right\}>\mathrm{C}_{\mathrm{th}}\left(1+\gamma_{4}+\gamma_{3}\right), \gamma_{1}>\gamma_{3}\right) \\
& =1-\left[\operatorname{Pr}\left(1+\min \left\{\gamma_{1}, \gamma_{2}\right\}>\mathrm{C}_{\mathrm{th}}\left(1+\gamma_{4}+\gamma_{3}\right)\right)\right. \\
& \left.-\operatorname{Pr}\left(1+\min \left\{\gamma_{1}, \gamma_{2}\right\}>\mathrm{C}_{\mathrm{th}}\left(1+\gamma_{3}+\gamma_{4}\right), \gamma_{1}<\gamma_{3}\right)\right] \\
& =I_{11}+I_{12}-I_{11} \times I_{12},
\end{aligned}
$$

where the second identity comes from the fact $P(A B)=$ $P(A)-P(A \bar{B})$. Notice that the third term of the second identity is equal to zero, which has been proved in the derivation of Eq.(7). By using [14, Eq. (3.381.4)], $I_{1 \beta}(\beta \in\{1,2\})$ is given by

$$
\begin{aligned}
& I_{1 \beta}=\sum_{j=1}^{L} A_{\beta, j}\left(1-\sum_{p=0}^{m_{\beta}-1} \frac{\zeta_{\beta, j}^{p} e^{-\zeta_{\beta, j}\left(C_{t h}-1\right)}}{p !} \sum_{q=0}^{p}\left(\begin{array}{l}
p \\
q
\end{array}\right) \sum_{f=0}^{q}\left(\begin{array}{l}
q \\
f
\end{array}\right)\right. \\
& \left.\times \sum_{b=1}^{L} \sum_{d=1}^{L} \frac{K a_{3, b} a_{4, d} \Gamma\left(m_{3}+f\right) \Gamma\left(m_{4}+q-f\right)}{\left(\zeta_{\beta, j} C_{t h}+\zeta_{3, b}\right)^{m_{3}+f}\left(\zeta_{\beta, j} C_{t h}+\zeta_{4, d}\right)^{m_{4}+q-f}}\right),
\end{aligned}
$$

where $K=\left(C_{t h}-1\right)^{p-q} C_{t h}^{q}$.

B. Strictly Positive Secrecy Capacity

According to the definition in [9], SPSC is readily given by

$\mathrm{SPSC}=\operatorname{Pr}\left(\mathrm{C}_{\mathbf{s}}\left(\gamma_{\mathrm{D}}, \gamma_{\mathrm{E}}\right)>0\right)=\operatorname{Pr}\left(\min \left\{\gamma_{1}, \gamma_{2}\right\}>\left(\gamma_{3}+\gamma_{4}\right)\right)$

$$
=1-I_{21}-I_{22}+I_{21} \times I_{22} \text {. }
$$

In (10), $I_{2 \beta}(\beta \in\{1,2\})$ can be written as

$$
\begin{aligned}
& I_{2 \beta}=\sum_{j=1}^{L} A_{\beta, j}\left(1-\sum_{p=0}^{m_{\beta}-1} \frac{\zeta_{\beta, j}^{p}}{p !} \sum_{q=0}^{p}\left(\begin{array}{l}
p \\
q
\end{array}\right) \sum_{b=1}^{L} \sum_{d=1}^{L} a_{3, b} a_{4, d}\right. \\
& \left.\times \frac{\Gamma\left(m_{3}+q\right) \Gamma\left(m_{4}+p-q\right)}{\left(\zeta_{\beta, j}+\zeta_{3, b}\right)^{m_{3}+q}\left(\zeta_{\beta, j}+\zeta_{4, d}\right)^{m_{4}+p-q}}\right) .
\end{aligned}
$$

\section{Average Secrecy Capacity}

From (6), ASC can be expressed as

$$
\bar{C}_{s}=\int_{0}^{\infty} \int_{0}^{\infty} C_{s}\left(\gamma_{D}, \gamma_{E}\right) f\left(\gamma_{D}, \gamma_{E}\right) d \gamma_{D} d \gamma_{E}=\frac{1}{2}\left(H_{1}-H_{2}\right) \text {, }
$$

where $f\left(\gamma_{D}, \gamma_{E}\right)$ denotes the joint PDF of $\gamma_{D}$ and $\gamma_{E}$. Since $\gamma_{D}$ and $\gamma_{E}$ are not independent, the ASC analysis becomes more difficult compared to the analysis in [11][12]. To obtain $f\left(\gamma_{D}, \gamma_{E}\right)$, we can first compute the joint $\operatorname{CDF} F\left(\gamma_{D}, \gamma_{E}\right)$. In particular, at high SNR, we have

$$
\begin{aligned}
& F\left(\gamma_{D}, \gamma_{E}\right) \approx \operatorname{Pr}\left(\min \left\{\gamma_{1}, \gamma_{2}\right\}<\gamma_{\mathrm{D}}, \gamma_{4}+\min \left\{\gamma_{1}, \gamma_{3}\right\}<\gamma_{\mathrm{E}}\right) \\
& =\operatorname{Pr}\left(\min \left\{\gamma_{1}, \gamma_{2}\right\}<\gamma_{\mathrm{D}}\right) \\
& -\operatorname{Pr}\left(\min \left\{\gamma_{1}, \gamma_{2}\right\}<\gamma_{\mathrm{D}}, \gamma_{4}+\min \left\{\gamma_{1}, \gamma_{3}\right\}>\gamma_{\mathrm{E}}\right) \\
& =\operatorname{Pr}\left(\min \left\{\gamma_{1}, \gamma_{2}\right\}<\gamma_{\mathrm{D}}\right)-\operatorname{Pr}\left(\gamma_{4}+\min \left\{\gamma_{1}, \gamma_{3}\right\}>\gamma_{\mathrm{E}}\right) \\
& +\operatorname{Pr}\left(\min \left\{\gamma_{1}, \gamma_{2}\right\}>\gamma_{\mathrm{D}}, \gamma_{4}+\min \left\{\gamma_{1}, \gamma_{3}\right\}>\gamma_{\mathrm{E}}\right)
\end{aligned}
$$

since $\gamma_{D}>\gamma_{E}, \operatorname{Pr}\left(\min \left\{\gamma_{1}, \gamma_{2}\right\}>\gamma_{\mathrm{D}}, \gamma_{4}+\min \left\{\gamma_{1}, \gamma_{3}\right\}>\gamma_{\mathrm{E}}\right)=$ $\operatorname{Pr}\left(\gamma_{1}>\gamma_{\mathrm{D}}, \gamma_{2}>\gamma_{\mathrm{D}}, \gamma_{4}+\gamma_{1}>\gamma_{\mathrm{E}}, \gamma_{4}+\gamma_{3}>\gamma_{\mathrm{E}}\right)=\operatorname{Pr}\left(\gamma_{1}>\right.$ $\left.\gamma_{\mathrm{D}}\right) \operatorname{Pr}\left(\gamma_{2}>\gamma_{\mathrm{D}}\right) \operatorname{Pr}\left(\gamma_{4}+\gamma_{3}>\gamma_{\mathrm{E}}\right)=\left(1-\mathrm{F}_{\gamma_{1}}\left(\gamma_{\mathrm{D}}\right)\right)(1-$ $\left.\mathrm{F}_{\gamma_{2}}\left(\gamma_{\mathrm{D}}\right)\right)\left(1-\operatorname{Pr}\left(\gamma_{4}+\gamma_{3}<\gamma_{\mathrm{E}}\right)\right)$.

Then, we have

$$
\begin{aligned}
& U\left(\gamma_{E}\right)=\operatorname{Pr}\left(\gamma_{3}<\gamma_{\mathrm{E}}-\gamma_{4}\right)=\int_{0}^{\gamma_{\mathrm{E}}} \mathrm{F}_{\gamma_{3}}\left(\gamma_{\mathrm{E}}-\gamma_{4}\right) \mathrm{f}_{\gamma_{4}}\left(\gamma_{4}\right) \mathrm{d} \gamma_{4} \\
& =\sum_{b=1}^{L} A_{3, b}\left[F_{\gamma_{4}}\left(\gamma_{E}\right)-\sum_{c=1}^{L} \sum_{f=0}^{m_{3}-1} \sum_{\mu=0}^{f} Q\left(\gamma_{E}^{f-\mu} e^{-\zeta_{3, b} \gamma_{E}}\right.\right. \\
& \left.\left.-\sum_{n=0}^{m_{4}+\mu-1} \frac{\left(\zeta_{4, c}-\zeta_{3, b}\right)^{n}}{n !} \gamma_{E}^{f+n-\mu} e^{-\zeta_{4, c} \gamma_{E}}\right)\right]
\end{aligned}
$$


where $Q=\left(\begin{array}{l}f \\ \mu\end{array}\right) \frac{a_{4, c}(-1)^{u} \zeta_{3, b}^{f}}{f !\left(\zeta_{4, c}-\zeta_{3, b}\right)^{\left(m_{4}+\mu\right)}}\left(m_{4}+\mu-1\right)$ ! and $\zeta_{4, c} \neq \zeta_{3, b}$.

Taking the derivative of (14) with respect to $\gamma_{E}$, we have

$$
\begin{aligned}
& u\left(\gamma_{E}\right)=\sum_{b=1}^{L} A_{3, b}\left[f_{\gamma_{4}}\left(\gamma_{E}\right)-\sum_{c=1}^{L} \sum_{f=0}^{m_{3}-1} \sum_{\mu=0}^{f} Q((f-\mu)\right. \\
& \times \gamma_{E}^{f-\mu-1} e^{-\zeta_{3, b} \gamma_{E}}-\zeta_{3, b} \gamma_{E}^{f-\mu} e^{-\zeta_{3, b} \gamma_{E}}-\sum_{n=0}^{m_{4}+\mu-1} \frac{\left(\zeta_{4, c}-\zeta_{3, b}\right)^{n}}{n !} \\
& \left.\left.\times\left((f+n-\mu) \gamma_{E}^{f+n-\mu-1} e^{-\zeta_{4, c} \gamma_{E}}-\zeta_{4, c} \gamma_{E}^{f+n-\mu} e^{-\zeta_{4, c} \gamma_{E}}\right)\right)\right] .
\end{aligned}
$$

With (15) at hand and by taking the derivative of (13) with respect to $\gamma_{D}$ and $\gamma_{E}$, the joint PDF $f\left(\gamma_{D}, \gamma_{E}\right)$ can be obtained as

$$
\begin{aligned}
& f\left(\gamma_{D}, \gamma_{E}\right)=f_{\gamma_{1}}\left(\gamma_{D}\right) u\left(\gamma_{E}\right)+f_{\gamma_{2}}\left(\gamma_{D}\right) u\left(\gamma_{E}\right)- \\
& f_{\gamma_{1}}\left(\gamma_{D}\right) F_{\gamma_{2}}\left(\gamma_{D}\right) u\left(\gamma_{E}\right)-F_{\gamma_{1}}\left(\gamma_{D}\right) f_{\gamma_{2}}\left(\gamma_{D}\right) u\left(\gamma_{E}\right) .
\end{aligned}
$$

Substituting (16) into (12) yields

$$
H_{1}=H_{11}+H_{12}-H_{13}-H_{14}
$$

and

$$
H_{2}=H_{21}+H_{22}-H_{23}-H_{24},
$$

these integrals in (17) and (18) can be evaluated as follows.

From [16, Eq. (78)], we have

$$
\begin{aligned}
& H_{1 \psi}=\int_{0}^{\infty} \int_{0}^{\gamma_{D}} \ln \left(1+\gamma_{D}\right) f_{\gamma_{\psi}}\left(\gamma_{D}\right) u\left(\gamma_{E}\right) d \gamma_{E} d \gamma_{D} \\
& =\sum_{j=1}^{L} \sum_{b=1}^{L} \sum_{c=1}^{L} a_{\psi, j} A_{3, b}\left[Z_{1} \sum_{p=1}^{m_{\psi}} \frac{\Gamma\left(p-m_{\psi}, \zeta_{\psi, j}\right)}{\zeta_{\psi, j}^{p}}-\sum_{s=0}^{m_{4}-1} Z_{2}\right. \\
& \times \sum_{p=1}^{m_{\psi}+s} \frac{\Gamma\left(p-m_{\psi}-s, \xi_{2}\right)}{\xi_{2}^{p}}-\sum_{f=0}^{m_{3}-1} \sum_{\mu=0}^{f} Q\left(\sum_{p=1}^{z_{0}} \frac{\Gamma\left(p-z_{0}, \xi_{1}\right)}{\xi_{1}^{p}}\right. \\
& \left.\left.\times Z_{3}-\sum_{n=0}^{m_{4}+\mu-1} Z_{4} \sum_{p=1}^{z_{0}+n} \frac{\Gamma\left(p-z_{0}-n, \xi_{2}\right)}{\xi_{2}^{p}}\right)\right]
\end{aligned}
$$

where $\psi \in\{1,2\}, \Gamma(\alpha, x)$ is the upper incomplete gamma function, $z_{0}=m_{\psi}+f-\mu, Z_{1}=A_{4, c}\left(m_{\psi}-1\right) ! e^{\zeta_{\psi, j}}$, $Z_{2}=\frac{A_{4, c} \zeta_{4, c}^{s}\left(m_{\psi}+s-1\right) !}{s e^{-\left(\zeta_{\psi, j}+\zeta_{4, c}\right)}}, Z_{3}=\left(z_{0}-1\right) ! e^{\zeta_{\psi, j}+\zeta_{3, b}}, Z_{4}=$ $\frac{\left(\zeta_{4, c}-\zeta_{3, b}\right)^{\frac{n}{2} \cdot e^{-}\left(z_{0}+n-1\right) !}}{n ! e^{-\left(\zeta_{\psi}, j+\zeta_{4, c}\right)}}, \xi_{1}=\zeta_{\psi, j}+\zeta_{3, b}$, and $\xi_{2}=\zeta_{\psi, j}+\zeta_{4, c}$.

Let $(i, t)$ denote the two pairs $(1,2)$ and $(2,1)$. Then, we have

$$
\begin{aligned}
& X\left(\gamma_{i}, \gamma_{t}\right)=\int_{0}^{\infty} \int_{0}^{\gamma_{D}} \ln \left(1+\gamma_{D}\right) f_{\gamma_{i}}\left(\gamma_{D}\right) F_{\gamma_{t}}\left(\gamma_{D}\right) u\left(\gamma_{E}\right) d \gamma_{E} d \gamma_{D} \\
& =\sum_{g=1}^{L} A_{t, g}\left[H_{1 i}-\sum_{j=1}^{L} \sum_{b=1}^{L} \sum_{c=1}^{L} \sum_{q=0}^{m_{t}-1} Z_{4}\left(\sum_{p=1}^{z_{1}}\left(z_{1}-1\right) !\right.\right. \\
& \times \frac{A_{4, c} \Gamma\left(p-z_{1}, \zeta_{i, j}+\zeta_{t, g}\right)}{e^{-\left(\zeta_{i, j}+\zeta_{t, g}\right)}\left(\zeta_{t, g}+\zeta_{i, j}\right)^{p}}-\sum_{s=0}^{m_{4}-1} A_{4, c}\left(z_{1}+s-1\right) ! \\
& \times \sum_{p=1}^{z_{1}+s} \frac{\Gamma\left(p-z_{1}-s, \xi_{4}\right)}{e^{-\xi_{4}} \xi_{4}^{p}}-\sum_{f=0}^{m_{3}-1} \sum_{\mu=0}^{f} Q\left(\sum_{p=1}^{z_{2}}\left(z_{2}-1\right) ! Z_{5}\right. \\
& \left.\left.\left.-\sum_{n=0}^{m_{4}+\mu-1} \sum_{p=1}^{z_{2}+n} \frac{\left(\zeta_{4, c}-\zeta_{3, b}\right)^{n} \Gamma\left(p-z_{2}-n, \xi_{4}\right)}{n ! e^{-\xi_{4}} \xi_{4}^{p}}\right)\right)\right]
\end{aligned}
$$

where $z_{1}=m_{i}+q, z_{2}=m_{i}+q+f-\mu, \xi_{3}=\zeta_{i, j}+\zeta_{t, g}+$ $\zeta_{3, b}, \xi_{4}=\zeta_{i, j}+\zeta_{t, g}+\zeta_{4, c}, Z_{4}=\frac{a_{i, j} A_{3, b} \zeta_{t, g}^{q}}{q !}, Z_{5}=\frac{\Gamma\left(p-z_{2}, \xi_{3}\right)}{e^{-\xi_{3} \xi_{3}^{p}}}$. Therefore, we easily observe that $H_{13}=X\left(\gamma_{1}, \gamma_{2}\right)$ and $H_{14}=$ $X\left(\gamma_{2}, \gamma_{1}\right)$.

Similar to the analysis for $H_{11}$ and using [14, Eq. (3.381.3)] and [14, Eq. (8.352.2)], we have

$$
\begin{aligned}
& H_{2 \psi}=\int_{0}^{\infty} \int_{\gamma_{E}}^{\infty} \ln \left(1+\gamma_{E}\right) u\left(\gamma_{E}\right) f_{\gamma_{\psi}}\left(\gamma_{D}\right) d \gamma_{D} d \gamma_{E} \\
& =\sum_{j=1}^{L} \sum_{g=1}^{L} \sum_{b=1}^{L} \sum_{c=1}^{L} \sum_{l=0}^{m_{\psi}-1} Z_{6}\left[\sum_{p=1}^{z_{3}} \frac{a_{4, c}\left(z_{3}-1\right) ! \Gamma\left(p-z_{3}, \xi_{2}\right)}{e^{-\xi_{2}} \xi_{2}^{p}}\right. \\
& -\sum_{f=0}^{m_{3}-1} \sum_{\mu=0}^{f} Q\left((f-\mu) \sum_{p=1}^{z_{4}} \frac{\left(z_{4}-1\right) ! \Gamma\left(p-z_{4}, \xi_{1}\right)}{e^{-\xi_{1}} \xi_{1}^{p}}\right. \\
& -\zeta_{3, b} \sum_{p=1}^{z_{4}+1} \frac{z_{4} ! \Gamma\left(p-z_{4}-1, \xi_{1}\right)}{e^{-\xi_{1}} \xi_{1}^{p}}-\sum_{n=0}^{m_{4}+\mu-1} \frac{\left(\zeta_{4, c}-\zeta_{3, b}\right)^{n}}{n !} \\
& \times\left((f+n-\mu) \sum_{p=1}^{z_{4}+n} \frac{\left(z_{4}+n-1\right) ! \Gamma\left(p-z_{4}-n, \xi_{2}\right)}{e^{-\xi_{2} \xi_{2}^{p}}}\right. \\
& \left.\left.-\zeta_{4, c} \sum_{p=1}^{z_{4}+n+1} \frac{\left(z_{4}+n\right) ! \Gamma\left(p-z_{4}-n-1, \xi_{2}\right)}{e^{-\xi_{2}} \xi_{2}^{p}}\right)\right]
\end{aligned}
$$

where $Z_{6}=\frac{\zeta_{\psi, j}^{l}}{l !}\left(m_{\psi}-1\right) ! \zeta_{\psi, j}^{-m_{\psi}} a_{\psi, j} A_{3, b}, z_{3}=m_{4}+l, z_{4}=$ $l+f-\mu$.

Like the analysis for $H_{13}$ and $H_{14}$, we can obtain $H_{23}=$ $Y\left(\gamma_{1}, \gamma_{2}\right)$ and $H_{24}=Y\left(\gamma_{2}, \gamma_{1}\right)$, where $Y\left(\gamma_{i}, \gamma_{t}\right)$

$$
\begin{aligned}
& Y\left(\gamma_{i}, \gamma_{t}\right)=\int_{0}^{\infty} \int_{\gamma_{E}}^{\infty} \ln \left(1+\gamma_{E}\right) u\left(\gamma_{E}\right) f_{\gamma_{i}}\left(\gamma_{D}\right) F_{\gamma_{t}}\left(\gamma_{D}\right) d \gamma_{D} d \gamma_{E} \\
& =\sum_{g=1}^{L} A_{t, g}\left[H_{2 i}-\sum_{j=1}^{L} \sum_{b=1}^{L} \sum_{d=1}^{L} \sum_{q=0}^{m_{t}-1 m_{i}+q-1} \sum_{v=0}^{z_{5}} Z_{7}\left(\sum_{p=1}^{a_{4}} a_{4, c}\right.\right. \\
& \times \frac{\left(z_{5}-1\right) ! \Gamma\left(p-z_{5}, \xi_{4}\right)}{e^{-\xi_{4}} \xi_{4}^{p}}-\sum_{f=0}^{m_{3}-1} \sum_{\mu=0}^{f} Q\left(\sum_{p=1}^{z_{6}} \frac{\Gamma\left(p-z_{6}, \xi_{3}\right)}{e^{-\xi_{3} \xi_{3}^{p}}}\right. \\
& \times(f-\mu)\left(z_{6}-1\right) !-\zeta_{3, b} \sum_{p=1}^{z_{6}+1} \frac{z_{6} ! \Gamma\left(p-z_{6}-1, \xi_{3}\right)}{e^{-\xi_{3} \xi_{3}^{p}}} \\
& -\sum_{n=0}^{m_{4}+\mu-1} \frac{\left(\zeta_{4, c}-\zeta_{3, b}\right)^{n}}{n !}\left((f+n-\mu) \sum_{p=1}^{z_{6}+n} Z_{8}\right. \\
& \left.\left.\left.-\zeta_{4, c} \sum_{p=1}^{z_{6}+n+1} \frac{\left(z_{6}+n\right) ! \Gamma\left(p-z_{6}-n-1, \xi_{4}\right)}{e^{-\xi_{4}} \xi_{4}^{p}}\right)\right)\right],
\end{aligned}
$$

where $Z_{7}=\frac{a_{i, j} A_{3, b} \zeta_{t, g}^{q}\left(m_{i}+q-1\right) !}{\left(\zeta_{i, j}+\zeta_{t, j}\right)^{\left(m_{i}+q-v\right)} q ! v !}, z_{5}=m_{4}+v, z_{6}=v+$

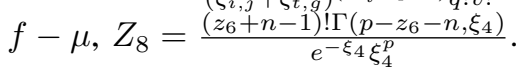

\section{NumericAl Results AND CONCLUSIONS}

In this section, some numerical results for the secrecy performance of a relay system suffering from the $K_{G}$ fading are presented. For comparison, we also present the MonteCarlo simulation to verify our analysis. From these figures, it is obviously shown that the analytical results match perfectly with their corresponding simulation results at mediate and 


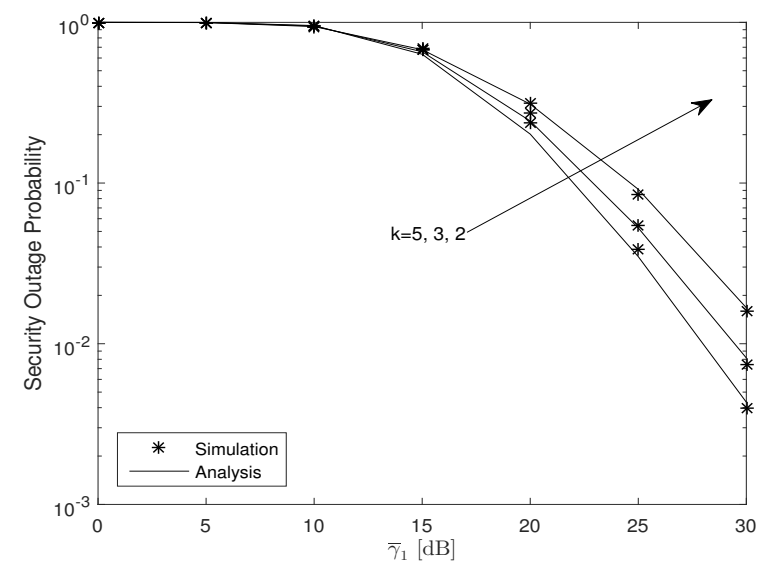

Fig. 1. SOP versus $\bar{\gamma}_{1}$ for various values of $k$ when $m_{1}=m_{2}=m_{3}=$ $m_{4}=2, \bar{\gamma}_{3}=10 \mathrm{~dB}, \bar{\gamma}_{4}=10 \mathrm{~dB}$, and $R_{t h}=0.01$.

high SNR regimes. Without loss of generality, we assume $k_{1}=k_{2}=k_{3}=k_{4}=k, L=5$, and the number of channel realizations is set to $10^{4}$.

In Fig. 1, we plot the SOP versus $\bar{\gamma}_{1}$ for different values of $k$ when $\bar{\gamma}_{2}=\bar{\gamma}_{1}$. From Fig. 1, we can find that increasing the value of $k$ results in improving the secrecy outage performance at high SNR. The reason is that the the severity of the shadowing degrades as increasing $k$.

We plot the SPSC performance versus $\bar{\gamma}_{1}$ for different $k$ when $\bar{\gamma}_{2}=\bar{\gamma}_{1}$ in Fig. 2. Also, a similar observation can be found as in Fig. 1. Fig. 3 demonstrates the ASC versus $\bar{\gamma}_{1}$ for different values of $k$ when $\bar{\gamma}_{2}=\bar{\gamma}_{1}$. Similarly, increasing $k$ results in enhancing the ASC at high SNR.

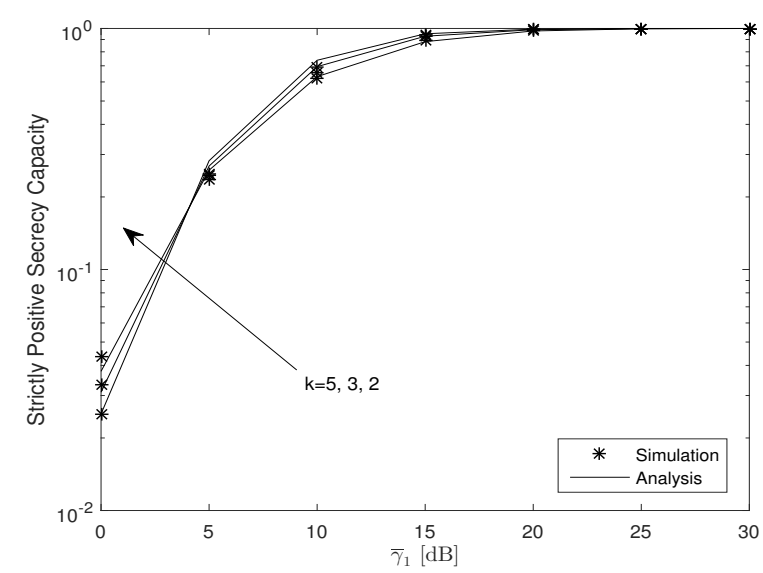

Fig. 2. SPSC versus $\bar{\gamma}_{1}$ for various values of $k$ when $m_{1}=m_{2}=m_{3}=$ $m_{4}=2, \bar{\gamma}_{3}=1 \mathrm{~dB}$, and $\bar{\gamma}_{4}=1 \mathrm{~dB}$.

\section{REFERENCES}

[1] Y. Liu, H.-H. Chen, and L. Wang, "Physical layer security for next generation wireless networks: Theories, technologies, and challenges," IEEE Commun. Surveys Tut., vol. 19, no. 1, pp. 347-376, Feb./Mar. 2017.

[2] Y. Zou, J. Zhu, X. Wang, and V. Leung, "Improving physical-layer security in wireless communications using diversity techniques," IEEE Netw., vol. 29, no. 1, pp. 42-48, Jan./Feb. 2015.

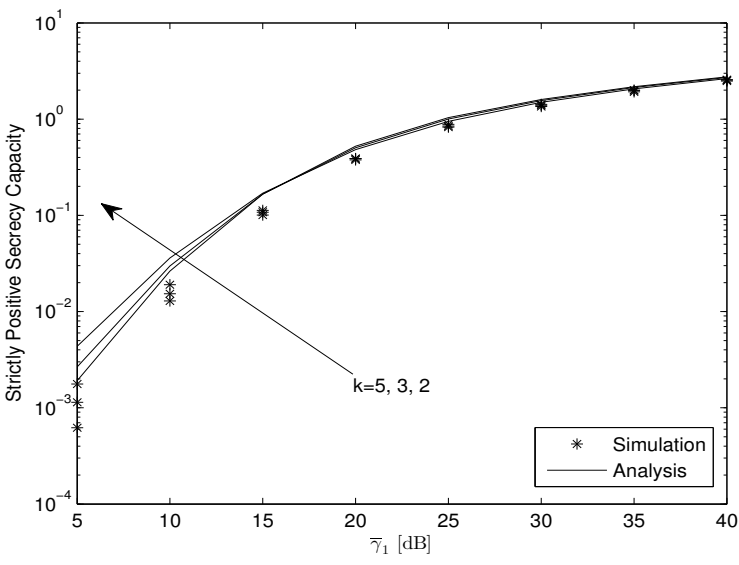

Fig. 3. ASC versus $\bar{\gamma}_{1}$ for various values of $k$ when $m_{1}=m_{2}=m_{4}=2$, $m_{3}=1, \bar{\gamma}_{3}=10 \mathrm{~dB}$, and $\bar{\gamma}_{4}=10 \mathrm{~dB}$.

[3] Y. Zou, X.Wang, and W. Shen, "Optimal relay selection for physical-layer security in cooperative wireless networks," IEEE J. Sel. Areas Commun., vol. 31, no. 10, pp. 2099-2111, Oct. 2013.

[4] L. Fan, X. Lei, T. Q. Duong, M. Elkashlan, and G. Karagiannidis, "Secure multiuser communications in multiple amplify-and-forward relay networks," IEEE Trans. Commun., vol. 62, no. 9, pp. 3299-3310, Sept. 2014.

[5] Y. Huang, J. Wang, C. Zhong, T. Q. Duong, and G. K. Karagiannidis, "Secure transmission in cooperative relaying networks with multiple antennas," IEEE Trans. Wireless Commun., vol. 15, no. 10, pp. 68436856, Oct. 2016.

[6] Y. Feng, S. Yan, Z. Yang,N. Yang and W. Zhu, "TAS-Based incremental hybrid decode-amplify-forward relaying for physical layer security enhancement," IEEE Trans. Wireless Commun., vol. 65, no. 9, pp. 38763891, Sep. 2017.

[7] M. Z. I. Sarkar and T. Ratnarajah, "Secrecy capacity over log-normal fading channel with diversity combining techniques," in 2013 IEEE Wireless Communications and Networking Conference (WCNC), April 2013, pp. 2457-2461.

[8] X. Liu, "Probability of strictly positive secrecy capacity of the weibull fading channel," in Proc. IEEE GLOBECOM, Atlanta, GA, USA, Dec. 2013, pp. 659-664.

[9] X. Liu, "Probability of strictly positive secrecy capacity of the ricianrician fading channel," IEEE Wireless Commun. Lett., vol. 2, no. 1, pp. 50-53, Feb. 2013.

[10] P. M. Shankar, "Error rates in generalized shadowed fading channels," Wireless Pers. Commun., vol. 28, no. 3, pp. 233-238, Feb. 2004.

[11] H. Lei C. Gao, I. S. Ansari, Y. Guo, G. Pan, and K. A. Qaraqe, "On physical-layer security over SIMO generalized-K fading channels," IEEE Trans. Veh. Technol., vol. 65, no. 9, pp. 7780-7785, Sep. 2016.

[12] H. Lei, H. Zhang, I. S. Ansari, C. Gao, Y. Guo, G. Pan, and K. A. Qaraqe, "Performance analysis of physical layer security over generalized-K fading channels using a mixture Gamma distribution," IEEE. Commun. Lett., vol. 20, no. 2, pp. 408-411, Feb. 2016.

[13] J. Jung, S. Lee, H. Park, and I. Lee, "Capacity and error probability analysis of diversity reception schemes over generalized-K fading channels using a mixture Gamma distribution," IEEE Trans. Wireless Commun., vol.13, no. 9, pp. 4721-4730, Sep. 2014.

[14] I. Gradshteyn and I. Ryzhik, Table of Integrals, Series and Products, 7th ed. San Diego, CA, USA: Academic, 2007.

[15] M. Bloch, J. Barros, M. R. D. Rodrigues, and S. W. McLaughlin, "Wireless information theoretic security," IEEE Trans. Inf. Theory., vol. 54, no. 6, pp. 2515-2534, June 2008.

[16] M.-S. Alouini and A. J. Goldsmith, "Capacity of Rayleigh fading channels under different adaptive transmission and diversity-combining techniques, "IEEE Trans. Veh. Technol., vol. 48, no. 4, pp. 1165-1181, Jul. 1999. 